\title{
Modal Operators in Intuitionistic Fuzzy Matrices
}

\author{
P. Murugadas \\ Assistant Professor \\ Mathematics Section, FEAT \\ Annamalai University, \\ Annamalainagar, India.
}

\author{
S.Sriram \\ Professor \\ Mathematics Section, FEAT \\ Annamalai University, \\ Annamalainagar, India.
}

\author{
T.Muthuraji \\ Assistant Professor \\ Mathematics Section, FEAT \\ Annamalai University, \\ Annamalainagar, India.
}

\begin{abstract}
In this paper, we introduce modal operators for Intuitionistic Fuzzy Matrix and derive some results.
\end{abstract}

\section{Keywords:}

Intuitionistic Fuzzy Matrices(IFMs), Intuitionistic Fuzzy Set.

\section{INTRODUCTION}

In conventional fuzzy set, a membership function assigns to each element of the universe of discourse a number from the unit interval $[0,1]$ to indicate the degree of belongingness to the set under consideration. Since the emergence of fuzzy set theory by Zadeh[8], many new approaches and theories treating imprecision and uncertainty have been proposed. One phenomenal generalization is intuitionistic fuzzy set theory by Atanassov[1] which is characterized by two function expressing the degree of belongingness and the degree of nonbelongingness, respectively. This idea, which is a natural generalization of a standard fuzzy set, seems to be useful in modelling many real life situations. In this generalization he introduced some operators called (modal operators) which are meaningless in fuzzy set theory and found a promising direction of research. Im et al.,[2], Jeong and Lee Hong[3], Khan S. K, Pal M and Amiya K. Shyamal[5], Meenashi AR and Gandhimathi[4], Sriram and Murugadas[6,7]and several authors have studied Intuitionistic Fuzzy Matrices. In this paper we explore the modal operators to IFM and discuss some properties under max-min composition.

\section{PRELIMINARIES}

Definition 2.1. [1]An Intuitionistic Fuzzy Set(IFS) $A$ in $E$ (universal set) is defined as an object of the following form $A=\left\{\left\langle x, \mu_{A}(x), \nu_{A}(x)\right\rangle / x \in E\right\}$, where the functions: $\mu_{A}(x)$ : $E \rightarrow[0,1]$ and $\nu_{A}(x): E \rightarrow[0,1]$ define the membership and non-membership of the element $x \in E$ respectively and for every $x \in E: 0 \leq \mu_{A(x)}+\nu_{A}(x) \leq 1$.

For simplicity we consider the pair $\left\langle x, x^{\prime}\right\rangle$ as membership and nonmembership function of an IFS with $x+x^{\prime} \leq 1$.

Definition 2.2. [6] For $\left\langle x, x^{\prime}\right\rangle,\left\langle y, y^{\prime}\right\rangle \in I F S$, define $\left\langle x, x^{\prime}\right\rangle \vee\left\langle y, y^{\prime}\right\rangle=\left\langle\max \{x, y\}, \min \left\{x^{\prime}, y^{\prime}\right\}\right\rangle$

$\left\langle x, x^{\prime}\right\rangle \wedge\left\langle y, y^{\prime}\right\rangle=\left\langle\min \{x, y\}, \max \left\{x^{\prime}, y^{\prime}\right\}\right\rangle$

$\left\langle x, x^{\prime}\right\rangle^{c}=\left\langle x^{\prime}, x\right\rangle$. Here we can replace $\vee$ by + (the component wise addition) and $\wedge$ by $\circ$ (the component wise multiplication). For component wise multiplication even we can use $\left\langle x, x^{\prime}\right\rangle\left\langle y, y^{\prime}\right\rangle=$ $\left\langle\min \{x, y\}, \max \left\{x^{\prime}, y^{\prime}\right\}\right\rangle$.

Here $\left\langle x, x^{\prime}\right\rangle \geq\left\langle y, y^{\prime}\right\rangle$ means $x \geq y$ and $x^{\prime} \leq y^{\prime}$. And $\left\langle x, x^{\prime}\right\rangle<$ $\left\langle y, y^{\prime}\right\rangle$ means $x<y$ and $x^{\prime}>y^{\prime}$.

Definition 2.3. [6] An IFM is a matrix of pairs $A=$ $\left(\left\langle a_{i j}, a_{i j}^{\prime}\right\rangle\right)$ of non negative real numbers satisfying $a_{i j}+a_{i j}^{\prime} \leq 1$ for all $i, j$. The universal IFM $J=(\langle 1,0\rangle)$ for all entries and The zero matrix $O=(\langle 0,1\rangle)$ for all entries. Denote the set of all IFMs of order $m \times n$ by $\mathscr{F}_{m n}$ and square matrix of order $\mathrm{n}$ by $\mathscr{F}_{n}$.

Definition 2.4. [6] For IFMs $A=\left(\left\langle a_{i j}, a_{i j}^{\prime}\right\rangle\right)_{m \times n}, B=$ $\left(\left\langle b_{i j}, b_{i j}^{\prime}\right\rangle\right)_{m \times n}$ and $C=\left(\left\langle c_{i j}, c_{i j}^{\prime}\right\rangle\right)_{n \times p}$, define

$A \vee B=\left(\left\langle a_{i j}, a_{i j}^{\prime}\right\rangle \vee\left\langle b_{i j}, b_{i j}^{\prime}\right\rangle\right)=A+B$

$A \wedge B=\left(\left\langle a_{i j}, a_{i j}^{\prime}\right\rangle \wedge\left\langle b_{i j}, b_{i j}^{\prime}\right\rangle\right)=A \circ B$

$A C=\left(\bigvee_{k}\left(\left\langle a_{i k}, a_{i k}^{\prime}\right\rangle \wedge\left\langle c_{k j}, c_{k j}^{\prime}\right\rangle\right)\right)$,

$A \diamond C=\left(\bigwedge_{k}\left(\left\langle a_{i k}, a_{i k}^{\prime}\right\rangle \vee\left\langle c_{k j}, c_{k j}^{\prime}\right\rangle\right)\right)$,

$A^{T}=\left(\left\langle a_{j i}, a_{j i}^{\prime}\right\rangle\right), A^{c}=\left(\left\langle a_{i j}^{\prime}, a_{i j}\right\rangle\right)$.

Also we can write

$A C=\left(\left\langle\sum_{k=1}^{n} a_{i k} c_{k j}, \prod_{k=1}^{n}\left(a_{i k}^{\prime}+c_{k j}^{\prime}\right)\right\rangle\right)$

Definition 2.5. [1] For IFS $A$ Atanassov has defined the modal operators $\square$ and $\diamond$ in the following way.

$\square A=\left\{\left\langle\mu_{A}(x), 1-\mu_{A}(x)\right\rangle \mid x \in E\right.$, the Universal set $\}$ and $\diamond A=\left\{\left\langle 1-\nu_{A}(x), \nu_{A}(x)\right\rangle \mid x \in E\right\}$.

Proposition 2.6. [7] $(A \circ B)^{c}=A^{c}+B^{c}$ for $A, B \in \mathscr{F}_{m n}$.

Proposition 2.7. [7] $(A+B)^{c}=A^{c} \circ B^{c}$ for $A, B \in \mathscr{F}_{m n}$.

\section{RESULTS USING MODAL OPERATORS IN IFM}

In this section we define the operators $\square, \diamond$ for IFM and discuss the relation between this new operators.

Definition 3.1. For IFM $A$, define $\square A=\left(\left\langle a_{i j}, 1-a_{i j}\right\rangle\right)$ and $\diamond A=\left(\left\langle 1-a_{i j}^{\prime}, a_{i j}^{\prime}\right\rangle\right)$.

LEMMA 3.2

$1-\prod_{k=1}^{n}\left(a_{i k}+b_{k j}\right)=\sum_{k=1}^{n}\left(1-a_{i k}\right)\left(1-b_{k j}\right)$

Proof. Let $\prod_{k=1}^{n}\left(a_{i k}+b_{k j}\right)=a_{i l}$ for some $l$,

then $1-\prod_{k=1}^{n}\left(a_{i k}+b_{k j}\right)=1-a_{i l}$, 
$a_{i l} \geq b_{l j}$ and so $1-a_{i l} \leq 1-b_{l j}$.

Now $\sum_{k=1}^{n}\left(1-a_{i k}\right)\left(1-b_{k j}\right)=\left(1-a_{i l}\right)\left(1-b_{l j}\right)+\sum_{k \neq l}\left(1-a_{i k}\right)(1-$ $\left.b_{k j}\right)$

$=\left(1-a_{i l}\right)+\sum_{k \neq l}\left(1-a_{i k}\right)\left(1-b_{k j}\right)$

case 1.If $a_{i k}>a_{i l}$ and $b_{k j}<a_{i l}$

$1-a_{i k}<1-a_{i l}$ and $1-b_{k j}>1-a_{i l}>1-a_{i k} \Rightarrow\left(1-a_{i k}\right)(1-$ $\left.b_{k j}\right)=\left(1-a_{i k}\right)$.

Therefore (3.3) becomes

$\sum_{k=1}^{n}\left(1-a_{i k}\right)\left(1-b_{k j}\right)=\left(1-a_{i l}\right)+\left(1-a_{i k}\right)=\left(1-a_{i l}\right) \ldots$

From (3.2) and (3.4), (3.1) holds

case 2. If $a_{i k}<a_{i l}$ and $b_{k j}>a_{i l}$

$1-a_{i k}>1-a_{i l}, 1-b_{k j}<1-a_{i l}<1-a_{i k}$

Therefore $\left(1-a_{i k}\right)\left(1-b_{k j}\right)=\left(1-b_{k j}\right)$ and

$\sum_{k=1}^{n}\left(1-a_{i k}\right)\left(1-b_{k j}\right)=\left(1-a_{i l}\right)+\left(1-b_{k j}\right)=\left(1-a_{i l}\right) \ldots$

From (3.2) and (3.5), (3.1) holds. Similarly the following cases are also hold

case. 3 If $a_{i k}>a_{i l}$ and $b_{k j}>a_{i l}$.

case. 4 If $a_{i k}<a_{i l}$ and $b_{k j}<a_{i l}$.

LEMMA 3.3. $1-\sum_{k} a_{i k} b_{k j}=\prod_{k}\left(\left(1-a_{i k}\right)+\left(1-b_{k j}\right)\right) \ldots$

Proof. Set $\sum a_{i k} b_{k j}=a_{i l}$ for some $l$, then $a_{i l}<b_{l j}$ and so $1-a_{i l}>1-b_{l j}$

Therefore $1-\sum_{k} a_{i k} b_{k j}=1-a_{i l}$

Now $\prod_{k}\left(\left(1-a_{i k}\right)+\left(1-b_{k j}\right)\right)=\left(\left(1-a_{i l}\right)+\left(1-b_{l j}\right)\right) \prod_{k \neq l}((1-$ $\left.\left.a_{i k}\right)+\left(1-b_{k j}\right)\right)$

$=\left(1-a_{i l}\right) \prod_{k}\left(\left(1-a_{i k}\right)+\left(1-b_{k j}\right)\right)$

case. 1 If $a_{i k}>a_{i l}$ and $b_{k j}<a_{i l}$,

then $1-a_{i k}<1-a_{i l}$ and $1-b_{k j}>1-a_{i l}>1-a_{i k}$

Therefore $\prod_{k}\left(\left(1-a_{i k}\right)+\left(1-b_{k j}\right)\right)=\left(1-a_{i l}\right)\left(1-b_{k j}\right)=\left(1-a_{i l}\right)$ ...(3.9)

Thus (3.6) holds from (3.7)and (3.9).

case.2 If $a_{i k}<a_{i l}$ and $b_{k j}>a_{i l}$

then $1-a_{i k}>1-a_{i l}$ and $1-b_{k j}<1-a_{i l}<1-a_{i k}$

Therefore $\prod_{k}\left(\left(1-a_{i k}\right)+\left(1-b_{k j}\right)\right)=\left(1-a_{i l}\right)\left(1-a_{i k}\right)=\left(1-a_{i l}\right)$

...(3.10)

Thus (3.6) holds from (3.7) and (3.10). Similarly we can prove the other cases.

LEMmA 3.4. $1-\min \left\{a_{i j}, b_{i j}\right\}=\max \left\{1-a_{i j}, 1-b_{i j}\right\}$ ...(3.11)

(i.e) $1-a_{i j} b_{i j}=\left(1-a_{i j}\right)+\left(1-b_{i j}\right)$

PROOF. case 1 . If $a_{i j} \geq b_{i j}$, then $1-a_{i j} \leq 1-b_{i j}$

Therefore $1-a_{i j} b_{i j}=1-b_{i j}$ and $\left(1-a_{i j}\right)+\left(1-b_{i j}\right)=1-b_{i j}$. Hence (3.11) holds.

case 2. If $a_{i j} \leq b_{i j}$, then $1-a_{i j} \geq 1-b_{i j}$

Therefore $1-a_{i j} b_{i j}=1-a_{i j}$ and $\left(1-a_{i j}\right)+\left(1-b_{i j}\right)=1-a_{i j}$. Hence (3.11) holds.

LeMmA 3.5. $1-\max \left\{a_{i j}, b_{i j}\right\}=\min \left\{1-a_{i j}, 1-b_{i j}\right\}$ ...(3.12)

(i.e) $1-\left(a_{i j}+b_{i j}\right)=\left(1-a_{i j}\right)\left(1-b_{i j}\right)$

PROOF. case 1 . If $a_{i j} \geq b_{i j}$, then $1-a_{i j} \leq 1-b_{i j}$

Therefore $1-\left(a_{i j}+b_{i j}\right)=1-a_{i j}$ and $\left(1-a_{i j}\right)\left(1-b_{i j}\right)=1-a_{i j}$. Hence (3.12) holds. case 2. If $a_{i j} \leq b_{i j}$, then $1-a_{i j} \geq 1-b_{i j}$

Therefore $1-\left(a_{i j}+b_{i j}\right)=1-b_{i j}$ and $\left(1-a_{i j}\right)\left(1-b_{i j}\right)=1-b_{i j}$. Hence (3.12) holds.

LEMMA 3.6. For $A=\left(\left\langle a_{i j}, a_{i j}^{\prime}\right\rangle\right) \in \mathscr{F}_{m n},\left(\square A^{c}\right)^{c}=\diamond A$ and $\left(\diamond A^{c}\right)^{c}=\square A$.

Proof. $A^{c}=\left(\left\langle a_{i j}^{\prime}, a_{i j}\right\rangle\right)$. Therefore $\square A^{c}=\left(\left\langle a_{i j}^{\prime}, 1-\right.\right.$ $\left.\left.a_{i j}^{\prime}\right\rangle\right) \Rightarrow\left(\square A^{c}\right)^{c}=\left(\left\langle 1-a_{i j}^{\prime}, a_{i j}^{\prime}\right\rangle\right)=\diamond A$. Similarly $\diamond A^{c}=$ $\left(\left\langle 1-a_{i j}, a_{i j}\right\rangle\right) \Rightarrow\left(\diamond A^{c}\right)^{c}=\left(\left\langle a_{i j}, 1-a_{i j}\right\rangle\right)=\square A$.

The following propositions are straight forward from the definition of $\square A$ and $\diamond A$.

Proposition 3.7. For $A$ the Universal matrix $J$ and zero matrix $O$ in $\mathscr{F}_{m n}$

(i) $\square(\diamond J)=J$

(ii) $\diamond(\square J)=J$

(iii) $\square(\diamond O)=O$

(iv) $\diamond(\square O)=O$

(v) $\square(\diamond A)=\diamond A$

(vi) $\diamond(\square A)=\square A$

(vii) $\square \square A=\square A\left(\square^{2} A=\square A\right)$

(viii) $\diamond \diamond A=\diamond A\left(\diamond^{2} A=\diamond A\right)$

(ix) $\square O=O, \diamond O=O$

( (x) $\square J=J, \diamond J=J$.

Proposition 3.8. For $A, B \in \mathscr{F}_{m n}, \square A \circ \square B=\square\left(A^{c}+\right.$ $\left.B^{c}\right)^{c}$.

Proof. $A^{c}+B^{c}=$

$\left(\left\langle\max \left\{a_{i j}^{\prime}, b_{i j}^{\prime}\right\}, \min \left\{a_{i j}, b_{i j}\right\}\right\rangle\right) \Rightarrow$

$\left(A^{c}+B^{c}\right)^{c}=$

$\left(\left\langle\min \left\{a_{i j}, b_{i j}\right\}, \max \left\{a_{i j}^{\prime}, b_{i j}^{\prime}\right\}\right\rangle\right)$

Now $\square\left(A^{c}+B^{c}\right)^{c}=\left(\left\langle\min \left\{a_{i j}, b_{i j}\right\}, 1-\min \left\{a_{i j}, b_{i j}\right\}\right\rangle\right) \ldots(3.13)$

We have $\square A=\left(\left\langle a_{i j}, 1-a_{i j}\right\rangle\right), \square B=\left(\left\langle b_{i j}, 1-b_{i j}\right\rangle\right)$

Therefore $\square A \circ \square B=$

$\left(\left\langle\min \left\{a_{i j}, b_{i j}\right\}, \max \left\{1-a_{i j}, 1-b_{i j}\right\}\right\rangle\right)$

$=\left(\left\langle\min \left\{a_{i j}, b_{i j}\right\}, 1-\min \left\{a_{i j}, b_{i j}\right\}\right\rangle\right)$

(by Lemma (3.3)). Therefore $\square A \circ \square B=\square\left(A^{c}+B^{c}\right)^{c}$ by (3.13) and (3.14).

PROPOSITION 3.9. For $A, B \in \mathscr{F}_{m n}, \square A+\square B=\square\left(A^{c} \circ\right.$ $\left.B^{c}\right)^{c}$.

Proof. $A^{c} \circ B^{c}=$

$\left(\left\langle\min \left\{a_{i j}^{\prime}, b_{i j}^{\prime}\right\}, \max \left\{a_{i j}, b_{i j}\right\}\right\rangle\right) \Rightarrow$

$\left(A^{c} \circ B^{c}\right)^{c}=$

$\left(\left\langle\max \left\{a_{i j}, b_{i j}\right\}, \min \left\{a_{i j}^{\prime}, b_{i j}^{\prime}\right\}\right\rangle\right)$

Now $\square\left(A^{c} \circ B^{c}\right)^{c}=\left(\left\langle\max \left\{a_{i j}, b_{i j}\right\}, 1-\max \left\{a_{i j}, b_{i j}\right\}\right\rangle\right) \ldots(3.15)$

We have $\square A=\left(\left\langle a_{i j}, 1-a_{i j}\right\rangle\right)$,

$\square B=\left(\left\langle b_{i j}, 1-b_{i j}\right\rangle\right)$

Therefore $\square A+\square B=$

$\left(\left\langle\max \left\{a_{i j}, b_{i j}\right\}, \min \left\{1-a_{i j}, 1-b_{i j}\right\}\right\rangle\right)$

$=\left(\left\langle\max \left\{a_{i j}, b_{i j}\right\}, 1-\max \left\{a_{i j}, b_{i j}\right\}\right\rangle\right)($ by Lemma (3.4))...(3.16)

(by Lemma (3.4)). Therefore $\square A+\square B=\square\left(A^{c} \circ B^{c}\right)^{c}$ by (3.15) and (3.16).

In the dual fashion we can prove $\square A \circ \square B=\square\left(A^{c}+B^{c}\right)^{c}$.

Proposition 3.10. For $A, B \in \mathscr{F}_{m n}, \diamond A \circ \diamond B=\diamond\left(A^{c}+\right.$ $\left.B^{c}\right)^{c}$

ProOF. $A^{c}+B^{c}=$

$\left(\left\langle\max \left\{a_{i j}^{\prime}, b_{i j}^{\prime}\right\}, \min \left\{a_{i j}, b_{i j}\right\}\right\rangle\right) \Rightarrow$

$\left(A^{c}+B^{c}\right)^{c}=$

$\left(\left\langle\min \left\{a_{i j}, b_{i j}\right\}, \max \left\{a_{i j}^{\prime}, b_{i j}^{\prime}\right\}\right\rangle\right)$ 
Now $\diamond\left(A^{c}+B^{c}\right)^{c}=\left(\left\langle 1-\max \left\{a_{i j}^{\prime}, b_{i j}^{\prime}\right\}, \max \left\{a_{i j}^{\prime}, b_{i j}^{\prime}\right\}\right\rangle\right)$ ...(3.17)

We have $\diamond A=\left(\left\langle 1-a_{i j}^{\prime}, a_{i j}^{\prime}\right\rangle\right), \diamond B=\left(\left\langle 1-b_{i j}^{\prime}, b_{i j}^{\prime}\right\rangle\right)$

Therefore $\diamond A \circ \diamond B=\left(\left\langle\min \left\{1-a_{i j}^{\prime}, 1-b_{i j}^{\prime}\right\}, \max \left\{a_{i j}^{\prime}, b_{i j}^{\prime}\right\}\right\rangle\right)=$ $\left(\left\langle 1-\max \left\{a_{i j}^{\prime}, b_{i j}^{\prime}\right\}, \max \left\{a_{i j}^{\prime}, b_{i j}^{\prime}\right\}\right\rangle\right)$ (by Lemma (3.3)) ...(3.18) Therefore $\diamond A \circ \diamond B=\diamond\left(A^{c}+B^{c}\right)^{c}$ by (3.17) and (3.18). In the dual fashion we can prove the following proposition.

Proposition 3.11. For $A, B \in \mathscr{F}_{m n}, \diamond A+\diamond B=\diamond\left(A^{c} \circ\right.$ $\left.B^{c}\right)^{c}$.

Proposition 3.12. For $A, B \in \mathscr{F}_{m n}, \square(A \circ B)=\square A \circ \square B$.

PRoOF. $\square(A \circ B)=\left(\left\langle\min \left\{a_{i j}, b_{i j}\right\}, 1-\min \left\{a_{i j}, b_{i j}\right\}\right\rangle\right)=$ $\left(\left\langle\min \left\{a_{i j}, b_{i j}\right\}, \max \left\{1-a_{i j}, 1-b_{i j}\right\}\right\rangle\right)$ (by Lemma (3.3)) $=\square A \circ \square B$.

Similarly we can prove the following Propositions. $B)$

Proposition 3.13. For $A, B \in \mathscr{F}_{m n}, \square A+\square B=\square(A+$ $B)$

Proposition 3.14. For $A, B \in \mathscr{F}_{m n}, \diamond A+\diamond B=\diamond(A+$ $B)$.

Proposition 3.15. For $A, B \in \mathscr{F}_{m n}, \diamond A \circ \diamond B=\diamond(A \circ$

Proposition 3.16. For $A, B, C \in \mathscr{F}_{m n}, \square((A+B) \circ C)=$ $(\square A+\square B) \circ \square C$.

Proposition 3.17. For $A, B, C \in \mathscr{F}_{m n}, \square((A \circ B \circ B)+$ $C)=(\square A \circ \square B)+\square C$.

Proposition 3.18. For $A, B, C \in \mathscr{F}_{m n}, \diamond((A \circ B \circ B)+$ $C)=(\diamond A \circ \diamond B)+\diamond C$.

\section{RESULTS USING MAX-MIN PRODUCT}

Proposition 4.1. For $A, B \in \mathscr{F}_{m n}, \square(A B)=\square A \square B$.

PROOF. $A B=\left(\left\langle\sum_{k} a_{i k} b_{k j}, \prod_{k}\left(a_{i k}^{\prime}+b_{k j}^{\prime}\right)\right\rangle\right)$

$\Rightarrow \square(A B)=\left(\left\langle\sum_{k} a_{i k}^{k} b_{k j}, 1-\sum_{k}^{k} a_{i k} b_{k j}\right\rangle\right)$

Now $\square A \square B=\left(\left\langle\sum_{k} a_{i k} b_{k j}, \prod_{k}\left(\left(1-a_{i k}\right)+\left(1-b_{k j}\right)\right)\right\rangle\right)$

$=\left(\left\langle\sum_{k} a_{i k} b_{k j}, 1-\sum_{k}^{k} a_{i k} b_{k j}\right\rangle\right)$ (by Lemma (3.2))

From (4.1) and (4.2),

$\square(A B)=\square A \square B$.

Proposition 4.2. For $A, B \in \mathscr{F}_{m n}, \diamond(A B)=\diamond A \diamond B$.

PROOF. $A B=\left(\left\langle\sum_{k} a_{i k} b_{k j}, \prod_{k}\left(a_{i k}^{\prime}+b_{k j}^{\prime}\right)\right\rangle\right)$

$\Rightarrow \diamond(A B)=\left(\left\langle 1-\prod_{k}^{k}\left(a_{i k}^{\prime}+b_{k j}^{\prime}\right), \prod_{k}\left(a_{i k}^{\prime}+b_{k j}^{\prime}\right)\right\rangle\right)$

Now $\diamond A \diamond B=\left(\left\langle\sum_{k}^{k}\left(1-a_{i k}^{\prime}\right)\left(1-b_{k j}^{\prime}\right), \prod_{k}\left(a_{i k}^{\prime}+b_{k j}^{\prime}\right)\right\rangle\right)$

$=\left(\left\langle 1-\prod_{k}\left(a_{i k}^{\prime}+b_{k j}^{\prime}\right), \prod_{k}\left(a_{i k}^{\prime}+b_{k j}^{\prime}\right)\right\rangle\right)($ by Lemma (3.1))

From (4.3) and (4.4),

$\diamond(A B)=\diamond A \diamond B$.

Proposition 4.3. For $A, B, \in \mathscr{F}_{m n}$, and $C \in \mathscr{F}_{m n}$ $\square((A+B) C)=\square(A C+B C)=\square(A C)+\square(B C)=\square(A+$ $B) \square C$
Proof. $(A+B) C=\left(\left\langle\sum_{k}\left(a_{i k}+b_{i k}\right) c_{k j}, \prod_{k}\left(a_{i k}^{\prime} b_{i k}^{\prime}+c_{k j}^{\prime}\right)\right\rangle\right)$ $=\left(\left\langle\sum_{k}\left(a_{i k} c_{k j}+b_{i k} c_{k j}\right), \prod_{k}\left(\left(a_{i k}^{\prime}+c_{k j}^{\prime}\right)\left(b_{i k}^{\prime}+c_{k j}^{\prime}\right)\right\rangle\right)\right.$ $\square((A+B) C)=\left(\left\langle\sum_{k}\left(a_{i k} c_{k j}+b_{i k} c_{k j}\right), 1-\sum_{k}\left(a_{i k} c_{k j}+b_{i k} c_{k j}\right)\right\rangle\right)$ ...(4.5)

$\ddot{A C}+B C=\left(\left\langle\sum_{k} a_{i k} c_{k j}+\sum_{k} b_{i k} c_{k j}, \prod_{k}\left(a_{i k}^{\prime}+c_{k j}^{\prime}\right) \prod\left(b_{i k}^{\prime}+c_{k j}^{\prime}\right)\right\rangle\right)$ $=\left(\left\langle\sum_{k}\left(a_{i k} c_{k j}+b_{i k} c_{k j}\right), \prod_{k}\left(\left(a_{i k}^{\prime}+c_{k j}^{\prime}\right)\left(b_{i k}^{\prime}+c_{k j}^{\prime}\right)\right\rangle\right)\right.$

Therefore $\square(A C+B C)=\left(\left\langle\sum_{k}\left(a_{i k} c_{k j}+b_{i k} c_{k j}\right), 1-\sum_{k}\left(a_{i k} c_{k j}+\right.\right.\right.$ $\left.\left.\left.b_{i k} c_{k j}\right)\right\rangle\right)$

From (4.5), (4.6) and Proposition (3.9) $\square((A+B) C)=\square(A C+$ $B C)$

Similarly we can prove the following Proposition

Proposition 4.4. For $A \in \mathscr{F}_{m n}$, and $B, C \in \mathscr{F}_{n m}$ $\square(A(B+C))=\square(A B+A C)=\square(A B)+\square(A C)=$ $\square(A) \square(B+C)$.

The following Proposition can be proved analogically.

Proposition 4.5. For $A, B, \in \mathscr{F}_{m n}$, and $C \in \mathscr{F}_{m n}$ $\diamond((A+B) C)=\diamond(A C+B C)=\diamond(A C)+\diamond(B C)=\diamond(A+$ $B) . \diamond C$.

Also the following Proposition is trivial.

Proposition 4.6. (i) $\square(A B) \square C=\square A \square(B C)$ (ii) $\diamond(A B) \diamond C=\diamond A \diamond(B C)$ for any IFM of compatible order.

\section{CONCLUSION}

As we know fuzzy matrix theory play vital roles in fuzzy linear equation and finding $g$-inverse of fuzzy matrix. Analogous to this, most of the theory of fuzzy matrix has been extended to IFM. But, is it possible to decompose any IFM in to any composition of fuzzy matrices? The work for such a result is not yet started. If we are able to do this, many of the results which are true for matrices will also be true for IFM. We have a decomposition for IFM using the above said results and it will be published in future.

\section{REFERENCES}

[1] Atanassov K., Intuitionistic Fuzzy Sets, VII ITKR's Session, Sofia, June 1983.

[2] Im, Lee E. P and Park S. W.,The Determinant of square Intuitionistic Fuzzy Matrices, Far East Journal of Mathematical Sciences, Vol. 3 (5) (2001), 789-796.

[3] Jeong N. G and Hong-Youl Lee.,Canonical Form of Transitive Intuitionistic Fuzzy Matrices, Honam Mathematical Journal, Vol. 27 (4) (2005), 543-550.

[4] Meenakshi A.R, and Gandhimathi T., Intuitionistic Fuzzy Relational Equations, advances in Fuzzy Mathematics, Vol. 5 (3) (2010), 239-244.

[5] Khan S.K., Pal M., and Amiya K Shyamal., Intuitionistic Fuzzy Matrices,Notes on Intuitionistic Fuzzy Sets, Vol. 8 (2) (2002), 51-62.

[6] Sriram .S and Murugadas.P., Contribution to a study on Generalized Fuzzy Matrices, Ph.D Thesis Department of Mathematics, Annamalai University, July-2011.

[7] Sriram S and P. Murugadas.,On Semi-ring of Intuitionistic Fuzzy Matrices, Applied Mathematical Science, Vol. 4(23), (2010), 1099-1105 
[8] Zadah L.A., Fuzzy Sets, Journal of Information and Control, Vol.8(1965), 338-353. 